\title{
Squeeze Film Lubrication between Rough Poroelastic Rectangular Plates with Micropolar Fluid: A Special Reference to the Study of Synovial Joint Lubrication
}

\author{
N. B. Naduvinamani and G. K. Savitramma \\ Department of Mathematics, Gulbarga University, Gulbarga 585106, India \\ Correspondence should be addressed to N. B. Naduvinamani; naduvinamaninb@yahoo.co.in
}

Received 24 November 2012; Accepted 12 December 2012

Academic Editors: L. Bourithis and H. Zhu

Copyright (C) 2013 N. B. Naduvinamani and G. K. Savitramma. This is an open access article distributed under the Creative Commons Attribution License, which permits unrestricted use, distribution, and reproduction in any medium, provided the original work is properly cited.

\begin{abstract}
The effects of surface roughness and poroelasticity on the micropolar squeeze film behavior between rectangular plates in general and that of synovial joints in particular are presented in this paper. The modified Reynolds equation, which incorporates the randomized surface roughness structure as well as elastic nature of articular cartilage with micropolar fluid as lubricant, is derived. The load-carrying capacity and time of approach as functions of film thickness during normal articulation of joints are obtained by using Christensen stochastic theory for rough surfaces with the assumption that the roughness asperity heights are to be small compared to the film thickness. It is observed that the effect of surface roughness has considerable effects on lubrication mechanism of synovial joints.
\end{abstract}

\section{Introduction}

The study of mechanism of synovial joints has recently become an active area of scientific research. The human joint is a dynamically loaded bearing which employs articular cartilage as the bearing and synovial fluid as the lubricant. Once a fluid film is generated, squeeze film action is capable of providing considerable protection to the cartilage surface. The loaded bearing synovial joints of the human body are the shoulder, hip, knee, and ankle joints; such joints have a lower friction coefficient and negligible wear. Synovial fluid is a clear viscous fluid, a dialysate of plasma containing mucopolysaccharides. Synovial fluid usually exhibits a nonNewtonian shear thinning behavior. However, under high shear rates, the viscosity of synovial fluid approaches a constant value not much higher than that of water [1]. Therefore a Newtonian lubricant model has often been used for synovial fluid in lubrication modeling [2]. In this study the synovial fluid is modeled as non-Newtonian micropolar fluid.

Articular cartilage is poroelastic or biphasic consisting of both fluid and solid phases. The importance of the unique biphasic load-carrying characteristics of articular cartilage and fluid flow inside has been recognized in the lubrication of synovial joints such as weeping and boosted lubrication theories. A more general biphasic lubrication theory was subsequently proposed by Mow and Lai [3]. However, it was not until in the 1990s that the relation between friction and interstitial fluid pressurization was comprehensively studied [4-7]. A number of friction studies have been carried out under a wide range of tribological conditions to investigate the biphasic lubrication of articular cartilage. Under both start-up and reciprocating motions of a cartilage plug against a metallic counterface friction was found to increase with loading time [4]. The transient friction behavior observed was a direct result of the interstitial fluid pressurization and fluid load support, directly measured experimentally $[6,8]$. However, for a similar configuration but under cyclic loading, friction was found to be similar or even at a higher level [9]. The importance of the biphasic lubrication has also been studied by enzymatic treatment of articular cartilage altering the biphasic properties and fluid pressurization such as chondroitinase [10-13]. However, the results obtained have been contradictory. Pickard et al. [10] found no major differences 
in friction levels following chondroitinase treatment, while Kumar et al. [11] and Basalo et al. [12] showed a significant reduction.

All these studies were confined to the smooth cartilage surfaces of human knee. But Sayles et al. [14] revealed experimentally that cartilage surfaces are rough, and roughness height distribution is Gaussian in nature. This has motivated us to investigate the influence of roughness of cartilage surfaces in lubrication aspects of synovial joint. Christensen [15] developed the stochastic theory to understand the influence of surface roughness in hydrodynamic lubrication of bearings. Many researchers have used this theory to analyze the effect of surface roughness of various types of bearings. Naduvinamani et al. [16] have studied the problem of squeeze film lubrication between rough rectangular plates with couple stress fluid as lubricant. These investigations have not incorporated the poroelasticity of the bearing surface.

The squeeze film lubrication characteristics of micropolar fluid have been extensively studied in the literature. Agrawal et al. [17] studied the squeeze film and externally pressurized bearings lubricated with micropolar fluids and found that the time of approach is more for the micropolar fluids as compared to the corresponding Newtonian fluids. The analytical solution of the problem of squeeze film lubrication of micropolar fluid between two parallel plates (onedimensional) has been given by Bujurke et al. [18]

In the present paper, a theoretical study of combined effects of surface roughness and micropolar fluid in squeeze film lubrication between poroelastic rectangular plates is presented. For mathematical simplicity, the average of three layers of the cartilage is modeled as a single poroelastic layer.

\section{Mathematical Formulation of the Problem}

The geometry and coordinates of the problem are as shown in Figure 1. The squeezing flow of micropolar fluid between two rectangular surfaces is considered. The upper rough articular surface is approaching the lower smooth poroelastic matrix normally with a constant velocity $V(=\partial h / \partial t)$. The lubricant in the joint cavity is taken to be Eringen's [19] micropolar fluid. As the load-bearing area of the synovial knee joint is small, the two surfaces may be considered to be parallel under high loading conditions. The moving boundary is characterized by

$$
h=h(t)+h_{s}(x, z, \xi)
$$

where $h(t)$ represents the nominal smooth part of the film geometry and $h_{s}$ is part due to the surface asperities measured from the nominal level and is a randomly varying quantity of zero mean and $\xi$ is an index parameter determining a definite roughness parameter.

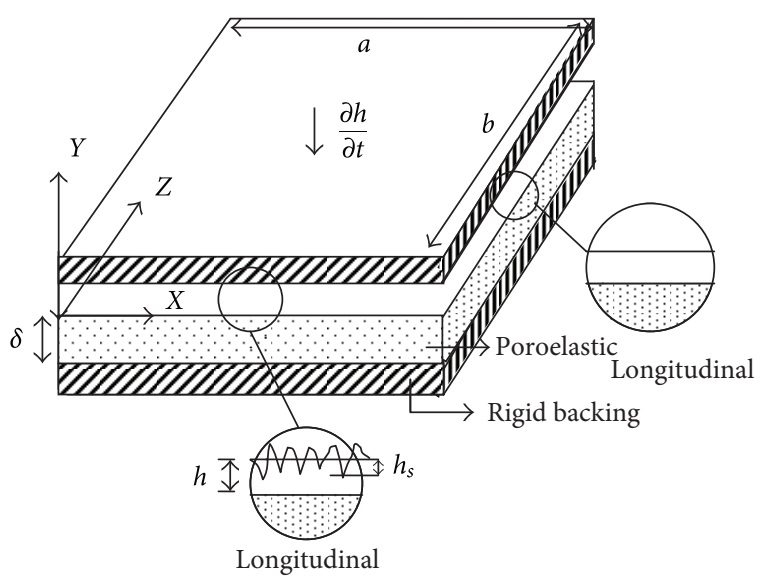

Figure 1: A geometry of simplified model for knee joint.

2.1. Basic Equations for Micropolar Fluids (Region-I). The basic equations for the flow of micropolar fluid in the film region in vectorial form are [19]

$$
\begin{gathered}
\frac{\partial \rho}{\partial t}+\nabla \cdot(\rho V)=0, \\
(\lambda+2 \mu+k) \nabla(\nabla \cdot V)-(\mu+k) \nabla \times(\nabla \times V)+k \nabla \times v \\
-\nabla \pi+\rho f=\rho\left[\frac{\partial V}{\partial t}-V \times(\nabla \times V)+\frac{1}{2} \nabla\left(V^{2}\right)\right] \\
(\alpha+\beta+\gamma) \nabla(\nabla \cdot V)-\gamma \nabla \times(\nabla \times V) \\
+k \nabla \times V-2 k v+\rho l=\rho j \dot{v} .
\end{gathered}
$$

For the three-dimensional steady motion of an incompressible micropolar fluid under the usual assumption of hydrodynamic lubrication with negligible body forces and body couples, the field equations (2) reduce to the following

\subsubsection{Conservation of Mass. Consider}

$$
\frac{\partial u}{\partial x}+\frac{\partial v}{\partial y}+\frac{\partial w}{\partial z}=0
$$

\subsubsection{Conservation of Linear Momentum. Consider}

$$
\begin{gathered}
\left(\mu+\frac{\chi}{2}\right) \frac{\partial^{2} u}{\partial y^{2}}+\chi \frac{\partial v_{2}}{\partial y}-\frac{\partial p}{\partial x}=0 \\
\frac{\partial p}{\partial y}=0 \\
\left(\mu+\frac{\chi}{2}\right) \frac{\partial^{2} w}{\partial y^{2}}+\chi \frac{\partial v_{1}}{\partial y}-\frac{\partial p}{\partial z}=0 .
\end{gathered}
$$




\subsubsection{Conservation of Angular Momentum. Consider}

$$
\begin{gathered}
\gamma \frac{\partial^{2} v_{1}}{\partial y^{2}}-2 \chi v_{1}-\chi \frac{\partial w}{\partial y}=0 \\
\gamma \frac{\partial^{2} v_{2}}{\partial y^{2}}-2 \chi v_{2}-\chi \frac{\partial u}{\partial y}=0
\end{gathered}
$$

where $u, v$, and $w$ are velocity components along $x, y$, and $z$ axes, respectively $v_{1}$ and $v_{2}$ are the micropolar velocity components in the $x$ and $z$ directions, respectively, and $p$ is the pressure in the film region.

2.2. Basic Equations for Poroelastic Region (Region-II). Following Torzilli and Mow [20] and Collins [21] the coupled equations of motion for deformable cartilage matrix and the mobile portion of the fluid contained in it can be written in the following form:

Matrix:

$$
\rho_{m} \frac{\partial^{2} \vec{U}}{\partial t^{2}}=\operatorname{div} \tau_{m}-\frac{1}{k^{*}}\left(\frac{\partial \vec{U}}{\partial t}-\vec{V}\right),
$$

Fluid:

$$
\rho_{f} \frac{D \vec{v}}{D t}=\operatorname{div} \tau_{f}+\frac{1}{k^{*}}\left(\frac{\partial \vec{U}}{\partial t}-\vec{V}\right),
$$

where $\rho_{m}$ and $\rho_{f}$ denote the densities of solid matrix and fluid, respectively, $\vec{U}$ is the corresponding displacement vector, $\vec{V}$ is the fluid velocity vector, $k^{*}$ is the permeability of the cartilage, and $D / D t$ denotes the material derivative. Equations (6) and (7) represent the force balance for the linear elastic solid and viscous fluid components, of the cartilage, respectively. In these equations, left hand terms denote the local forces (mass $\times$ acceleration) which are counterbalanced by right porous media driving force, respectively.

In fact these two equations may be viewed simply as a generalized form of Darcy's law for unsteady flow in a deformable porous medium in terms of the relative velocity $((d \vec{U} / d t)-\vec{V})$ between the moving cartilage and the fluid contained in its pores.

The classical stress tensor $\tau$ for a continuous homogeneous medium may be expressed for the matrix and fluid, respectively, as

$$
\begin{gathered}
\tau_{m}=p_{1} I+N^{1} e+A e I, \\
\tau_{f}=-p_{1} I+E e I,
\end{gathered}
$$

where $N^{1}, A$, and $E$ are the elastic parameters of the cartilage. After neglecting the inertia terms, addition of (6) and (7) eliminates the pressure and fluid velocity, and, thereafter, taking the divergence of the results yields the following Laplace equation:

$$
\nabla^{2} e=0
$$

where $e(=\operatorname{div} \vec{U})$ is known as the cartilage dilatation. Following Hori and Mockros [22] we characterize the cartilage dilatation by a sample similar linear equation in terms of corresponding average bulk modulus $K$, in the following form:

$$
e=e_{0}+\frac{p_{1}}{k^{*}}
$$

From (9) and (10) we get

$$
\nabla^{2} p_{1}=0
$$

The relevant boundary conditions for the velocity field are

$$
\begin{gathered}
u=w=0, \quad v=v_{n}, \quad v_{1}=v_{2}=0, \quad \text { at } y=0, \\
u=w=0, \quad v=-\frac{\partial h}{\partial t}, \quad v_{1}=v_{2}=0, \quad \text { at } y=h .
\end{gathered}
$$

\section{Solution of the Problem}

Solving (4) and (5) for the velocity components $u, v$, and $w$ and microrotation velocity components $v_{1}$ and $v_{2}$ with the respective boundary conditions given in (12) we get

$$
\begin{aligned}
u= & \frac{1}{\mu}\left[\frac{y^{2}}{2} \frac{\partial p}{\partial x}+C_{11} y\right] \\
& -\frac{2 N^{2}}{m}\left[C_{21} \sinh (m y)+C_{31} \cosh (m y)\right]+C_{41}, \\
v_{2}= & -\frac{1}{2 \mu}\left[y \frac{\partial p}{\partial x}+C_{11}\right]+\left[C_{21} \cosh (m y)+C_{31} \sinh (m y)\right] \\
v_{1}= & -\frac{1}{2 \mu}\left[y \frac{\partial p}{\partial z}+C_{12}\right]+\left[C_{22} \cosh (m y)+C_{32} \sinh (m y)\right] \\
w= & \frac{1}{\mu}\left[\frac{y^{2}}{2} \frac{\partial p}{\partial z}+C_{12} y\right] \\
& -\frac{2 N^{2}}{m}\left[C_{22} \sinh (m y)+C_{32} \cosh (m y)\right]+C_{42},
\end{aligned}
$$

where for $i=1,2$,

$$
\begin{aligned}
C_{1 i} & =2 \mu C_{2 i}, \\
C_{2 i} & =\frac{C_{3 i} \sinh (m h)-(h / 2 \mu)\left(\partial p / \partial x_{i}\right)}{1-\cosh (m h)}, \\
C_{3 i} & =\frac{h}{2 \mu} \frac{\partial p}{\partial x_{i}}\left[\frac{h}{2}(\cosh m h-1)+h-\frac{N^{2}}{m} \sinh m h\right] \frac{1}{C_{5}}, \\
C_{4 i} & =\frac{2 N^{2}}{m} C_{3 i}, \\
C_{5} & =h\left[\sinh (m h)-\frac{2 N^{2}}{m h}(\cosh m h-1)\right], \\
m & =\frac{N}{l}, \quad N=\left(\frac{\chi}{2 \mu+\chi}\right)^{1 / 2}, \quad l=\left(\frac{\gamma}{4 \mu}\right)^{1 / 2} .
\end{aligned}
$$


By neglecting inertia terms, (7) may be arranged in terms of relative velocity in the form

$$
\left(\bar{V}-\frac{d \vec{U}_{1}}{d t}\right)=-k^{*}\left(\nabla p_{1}-E \nabla e\right)
$$

and elimination of $e$ through (10) and (18) gives

$$
\left(\vec{V}-\frac{d \vec{U}_{1}}{d t}\right)=-k^{*} \nabla p_{1}\left(1-\frac{E}{K}\right) .
$$

The normal component of the relative fluid velocity at the cartilage surface is

$$
v_{n}=\left[-k^{*}\left(\frac{E}{K}-1\right) \frac{\partial p_{1}}{\partial y}\right]_{y=0} .
$$

Integrating (3) across the fluid film region and using the boundary conditions for $v$ given in (12) and also using the expressions (13) and (16) for $u$ and $w$, the modified Reynolds equation is obtained in the form

$$
\begin{gathered}
\frac{\partial}{\partial x}\left[f(N, l, h) \frac{\partial p}{\partial x}\right]+\frac{\partial}{\partial z}\left[f(N, l, h) \frac{\partial p}{\partial z}\right] \\
=\left[-12 \mu \frac{\partial h}{\partial t}+12 \mu k^{*}\left(1-\frac{E}{K}\right) \frac{\partial p_{1}}{\partial y}\right]_{y=0},
\end{gathered}
$$

where $f(N, l, h)=h^{3}+12 l^{2} h-6 N l h^{2} \cot (N h / 2 l)$.

3.1. Stochastic Reynolds Equation. In the context of rough surfaces, there are two types of roughness patterns which are of special interest. The one-dimensional longitudinal structure where the roughness has the form of long narrow ridges and valleys running in the $x$-direction and the onedimensional transverse structure where roughness striations are running in the $z$-direction in the form of long narrow ridges and valleys. However, the present study is restricted to one-dimensional longitudinal roughness since the one roughness structure can be obtained from others by just rotation of coordinate axes. For the one-dimensional longitudinal roughness pattern, the film thickness assumes the form

$$
h=h(t)+h_{s}(x, \xi) .
$$

Taking the expectation on both sides of (21) and simplifying them, the stochastic modified Reynolds type equation is obtained in the form

$$
\begin{aligned}
& \frac{\partial^{2} E(p)}{\partial x^{2}}+R \frac{\partial^{2} E(p)}{\partial z^{2}} \\
& \quad=\frac{1}{E[f(N, l, h)]}\left[-12 \mu \frac{\partial h}{\partial t}+12 \mu k\left(1-\frac{E}{K}\right) \frac{\partial p_{1}}{\partial y}\right]_{y=0},
\end{aligned}
$$

where

$$
\begin{gathered}
R=\left\{E[f(N, l, h)] * E\left[\frac{1}{f(N, l, h)}\right]\right\}^{-1}, \\
E[f(N, l, h)]=\frac{35}{32 c^{7}} \int_{-c}^{c} f(N, l, h)\left(c^{2}-h_{s}^{2}\right) d h_{s}, \\
E\left[\frac{1}{f(N, l, h)}\right]=\frac{35}{32 c^{7}} \int_{-c}^{c} \frac{\left(c^{2}-h_{s}^{2}\right)}{f(N, l, h)} d h_{s} .
\end{gathered}
$$

$E(\cdot)$ denotes the expectancy operator defined by

$$
E(\cdot)=\int_{-\infty}^{\infty}(\cdot) f\left(h_{s}\right) d h_{s} .
$$

$f\left(h_{s}\right)$ is the probability density function of the stochastic film thickness variable $h_{s}$. According to the Sayles et al. [14], the cartilage surfaces are rough and roughness height distribution is Gaussian in nature. Therefore, polynomial form which approximates the Gaussian distribution is chosen in the present study. Such a probability density function is

$$
f\left(h_{s}\right)= \begin{cases}\frac{35}{32 c^{7}}\left(c^{2}-h_{s}^{2}\right)^{3}, & -c \leq h_{s} \leq c \\ 0, & \text { elsewhere }\end{cases}
$$

where $c= \pm 3 \sigma$ and $\sigma$ is the standard deviation.

The relevant boundary conditions for $E(p)$ and $p_{1}$ are

(i) for the fluid film region

$$
E[p(x, z)]=0 \quad \text { at } x=0, a, z= \pm \frac{b}{2}
$$

(ii) for the poroelastic region

$$
\begin{gathered}
p_{1}(x, y, z)=0 \quad \text { at } x=0, a, z= \pm \frac{b}{2} \\
\frac{\partial p_{1}}{\partial y}=0 \quad \text { at } y=-\delta,
\end{gathered}
$$

(iii) at the interface

$$
p_{1}(x, y, z)=E[p(x, z)] \text { at } y=0,
$$

where $\delta$ is the cartilage layer thickness.

The solution of Laplace equation (11) with boundary conditions (28) and (29) is

$$
\begin{aligned}
p_{1} & (x, y, z) \\
& =\sum_{m=1}^{\infty} \sum_{n=1}^{\infty} C_{m n} \cosh \left[\gamma_{m n}(y-\delta)\right] \sin \left(\alpha_{m} x\right) \cos \left(\beta_{n} z\right),
\end{aligned}
$$

where

$$
\alpha_{m}=\frac{m \pi}{a}, \quad \beta_{n}=\frac{n \pi}{b}, \quad \gamma_{m n}=\left(\alpha_{m}^{2}+\beta_{n}^{2}\right)^{1 / 2},
$$


and the constants $C_{m n}$ are the Fourier coefficients to be determined.

Using the mean pressure continuity condition (30) in (31), we get

$$
E[p(x, z)]=\sum_{m=1}^{\infty} \sum_{n=1}^{\infty} C_{m n} \sin \left(\alpha_{m} x\right) \cos \left(\beta_{n} z\right) .
$$

On substituting (31) and (33) into (23) and using the orthogonality of eigen functions $\sin \left(\alpha_{m} x\right)$ and $\cos \left(\beta_{n} z\right)$, the Fourier coefficients $C_{m n}$ are obtained in the form

$$
\begin{aligned}
C_{m n}= & -192 \mu\left(\frac{\partial h}{\partial t}\right) \\
& \times\left(a b \alpha_{m} \beta_{n} E[f(N, l, h)]\right. \\
& \times\left\{\left(\alpha_{m}^{2}+R \beta_{n}^{2}\right)\right. \\
& \left.\left.-\frac{12 k^{*} \delta(1-(E / K)) \gamma_{m n} \tanh \left(\gamma_{m n} \delta\right)}{E[f(N, l, h)]}\right\}\right)^{-1},
\end{aligned}
$$

where $E[f(N, l, h)]=\left(35 / 32 c^{7}\right) \int_{-c}^{c} f(N, l, h)\left(c^{2}-h_{s}^{2}\right) d h_{s}$.

The load-carrying capacity of the squeeze film is obtained by integrating the averaged pressure field over the surface of the top plate

$$
E(W)=\int_{0}^{a} \int_{-b / 2}^{b / 2} E[p(x, z)] d z d x .
$$

The nondimensional mean instantaneous load-carrying capacity of the squeeze film is given by

$$
\begin{aligned}
& \bar{W}=-\frac{E(W) h_{0}^{3}}{\mu a^{2} b^{2} \dot{h}}=\frac{768 \bar{\delta} \sqrt{\lambda}}{\pi^{4}} \\
& \times \sum_{m=1,3, \ldots n=1,3, \ldots}^{\infty} \sum^{\infty}\left\{m ^ { 2 } n ^ { 2 } \left[\frac{\bar{\delta}}{\sqrt{\lambda}}\left(m^{2} \pi^{2}+R n^{2} \pi^{2} \lambda^{2}\right)\right.\right. \\
& \times E[f(N, \bar{l}, \bar{h})]-12 \psi\left(1-\frac{E}{K}\right) \\
&\left.\left.\times \bar{\gamma}_{m n} \tanh \left(\frac{\bar{\gamma}_{m n} \bar{\delta}}{\sqrt{\lambda}}\right)\right]\right\}^{-1},
\end{aligned}
$$

where

$$
\begin{gathered}
\bar{l}=\frac{l}{h_{0}}, \quad \bar{h}=\frac{h}{h_{0}}, \quad \bar{c}=\frac{c}{h_{0}}, \quad \lambda=\frac{a}{b}, \quad \bar{\delta}=\frac{\delta}{\sqrt{a b}}, \\
\bar{\gamma}_{m n}=\gamma_{m n} a, \quad \psi=\frac{k^{*} \delta}{h_{0}^{3}}, \quad \bar{k}=\frac{k^{*}}{h_{0}^{2}} \\
E[f(N, \bar{l}, \bar{h})]=\frac{1}{h_{0}^{3}} E[f(N, l, h)]
\end{gathered}
$$

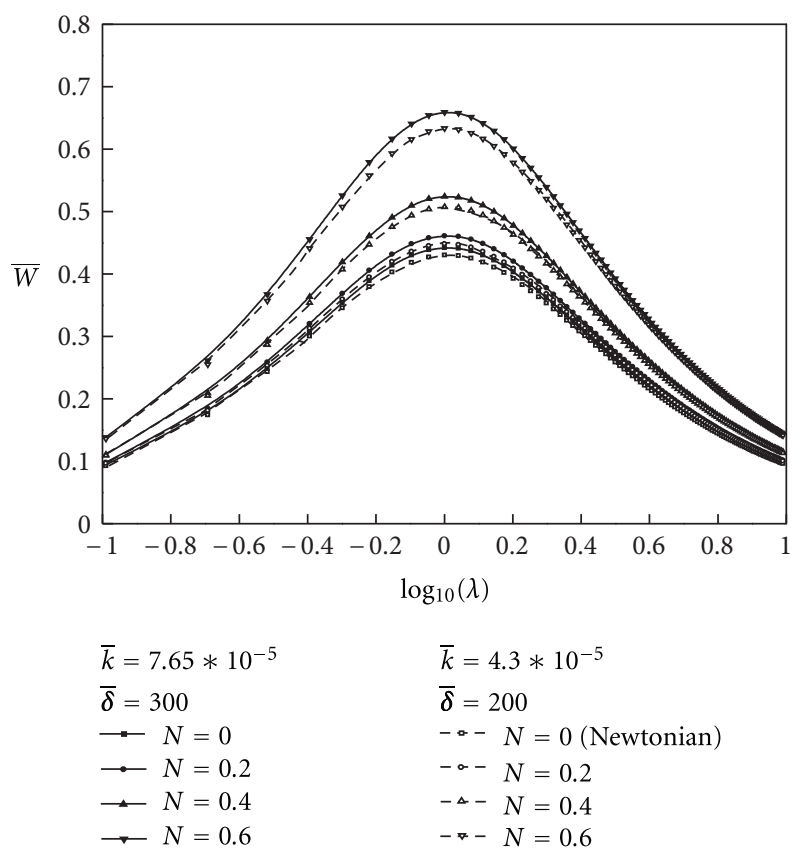

Figure 2: Variation of non-dimensional load $\bar{W}$ with $\log _{10}(\lambda)$ for different values of $N$ with $\bar{l}=0.2, E / K=0.3, \bar{c}=0.2$.

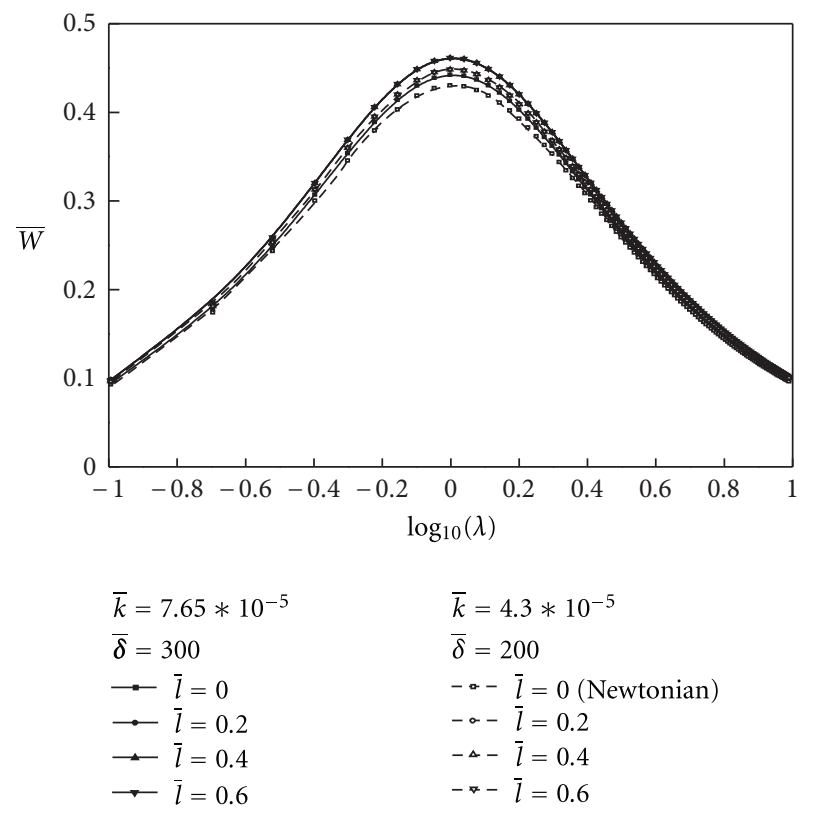

Figure 3: Variation of non-dimensional load $\bar{W}$ with $\log _{10}(\lambda)$ for different values of $\bar{l}$ with $N=0.2, E / K=0.3, \bar{c}=0.2$.

The film thickness at any time $t$ can be obtained by solving for the load as a function of time:

$$
t=\int_{0}^{t} E(W(T)) d T=\frac{-768 \mu a^{2} b^{2} \bar{\delta} \sqrt{\lambda}}{h_{0}^{2} \pi^{4}} \sum_{m=1,3, \ldots}^{\infty} \sum_{n=1,3, \ldots}^{\infty} I_{m n}
$$




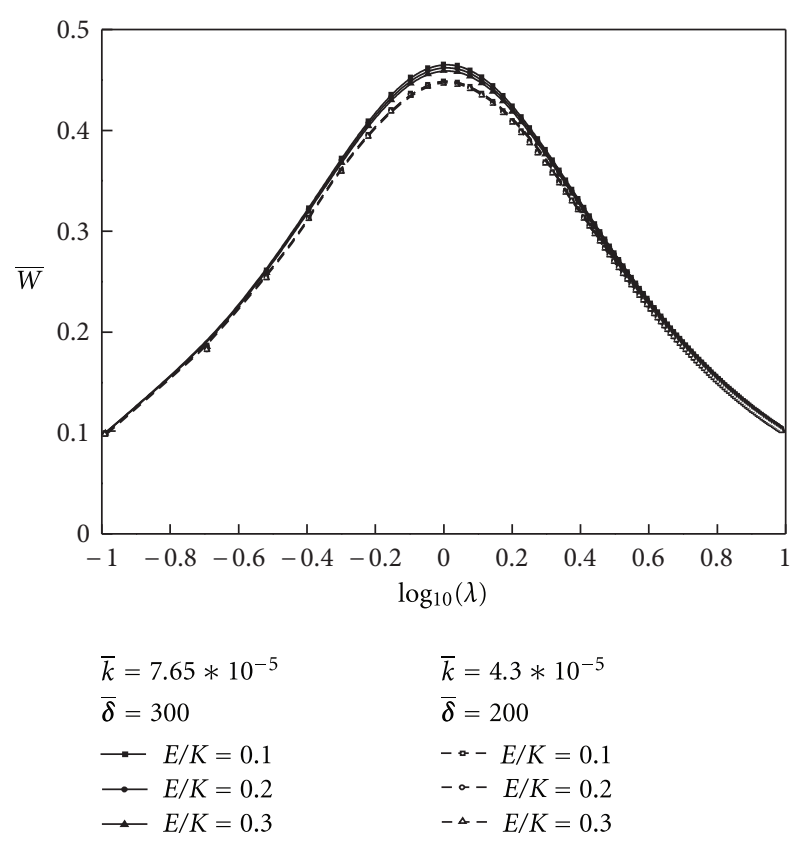

FIgURE 4: Variation of non-dimensional load $\bar{W}$ with $\log _{10}(\lambda)$ for different values of $E / K$ with $N=0.2, \bar{l}=0.2, \bar{c}=0.2$.

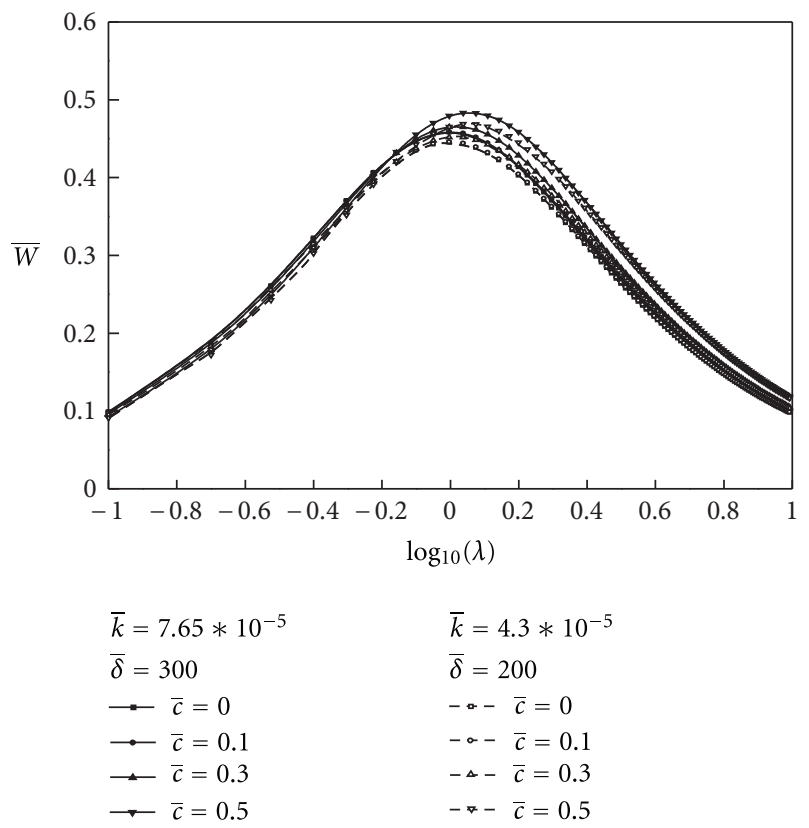

FIgURE 5: Variation of non dimensional load $\bar{W}$ with $\log _{10}(\lambda)$ for different values of $\bar{c}$ with $\bar{l}=0.2, N=0.2, E / K=0.3$.

where

$$
\begin{aligned}
I_{m n}=\int_{1}^{\bar{h}}\left\{m^{2} n^{2}\right. & {\left[\frac{\bar{\delta}}{\sqrt{\lambda}}\left(m^{2} \pi^{2}+\bar{R} n^{2} \pi^{2} \lambda^{2}\right) E[f(N, \bar{l}, \bar{h})]\right.} \\
& \left.\left.-12 \psi\left(1-\frac{E}{K}\right) \bar{\gamma}_{m n} \tanh \left(\frac{\bar{\gamma}_{m n} \bar{\delta}}{\sqrt{\lambda}}\right)\right]\right\}^{-1} \cdot d \bar{h}
\end{aligned}
$$

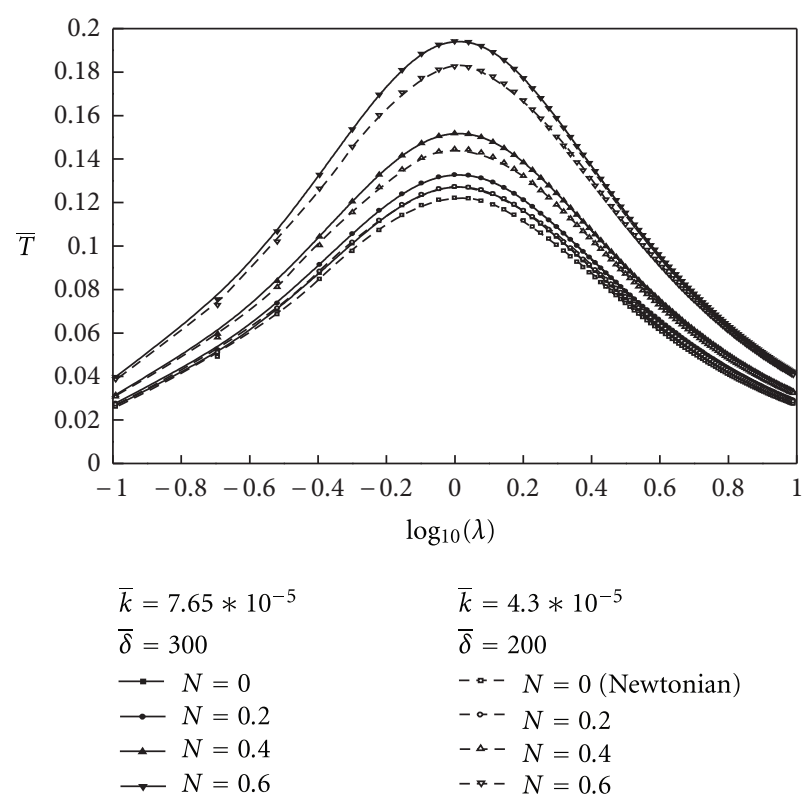

Figure 6: Variation of non-dimensional squeeze film time $\bar{T}$ with $\log _{10}(\lambda)$ for different values of $N$ with $\bar{l}=0.2, E / K=0.3, \bar{c}=0.2$.

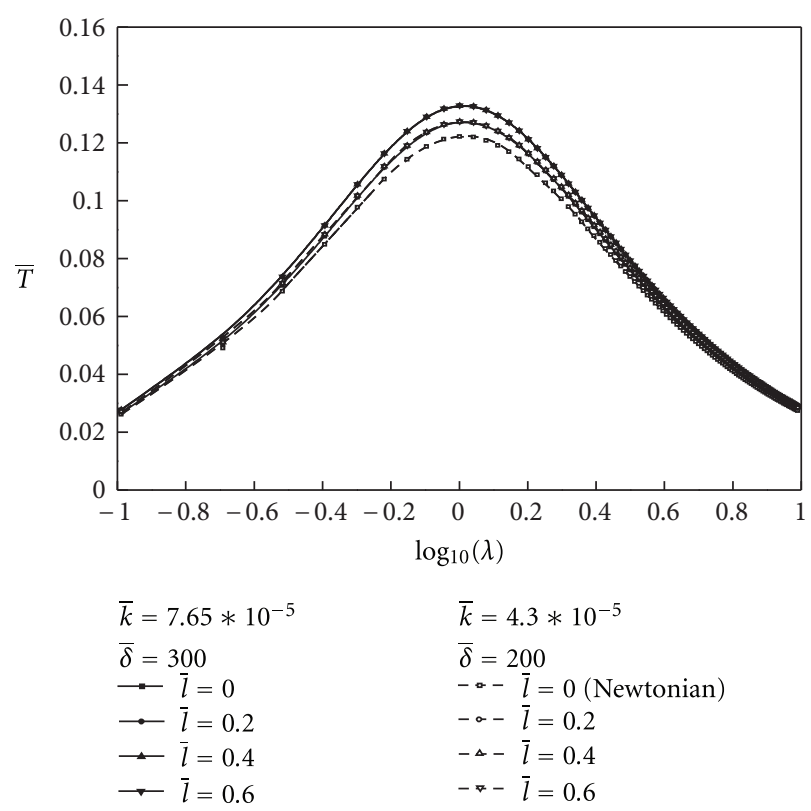

Figure 7: Variation of non-dimensional squeeze film time $\bar{T}$ with $\log _{10}(\lambda)$ for different values of $\bar{l}$ with $N=0.2, E / K=0.3, \bar{c}=0.2$.

The nondimensional squeeze film time is obtained as

$$
\bar{T}=-\frac{E(W(t)) h_{0}^{2}}{\mu a^{2} b^{2}}=\frac{768 \bar{\delta} \sqrt{\lambda}}{\pi^{4}} \sum_{m=1,3, \ldots n=1,3, \ldots}^{\infty} \sum_{m n}^{\infty}
$$

As $\bar{k} \rightarrow 0$ (i.e., $\psi \rightarrow 0$ ) and (36) and (40) reduce to the corresponding solid case studied by Sinha and Singh [23], the results of $\mathrm{Wu}$ [24] can be recovered in the limiting case of $N, \bar{l} \rightarrow 0$. 


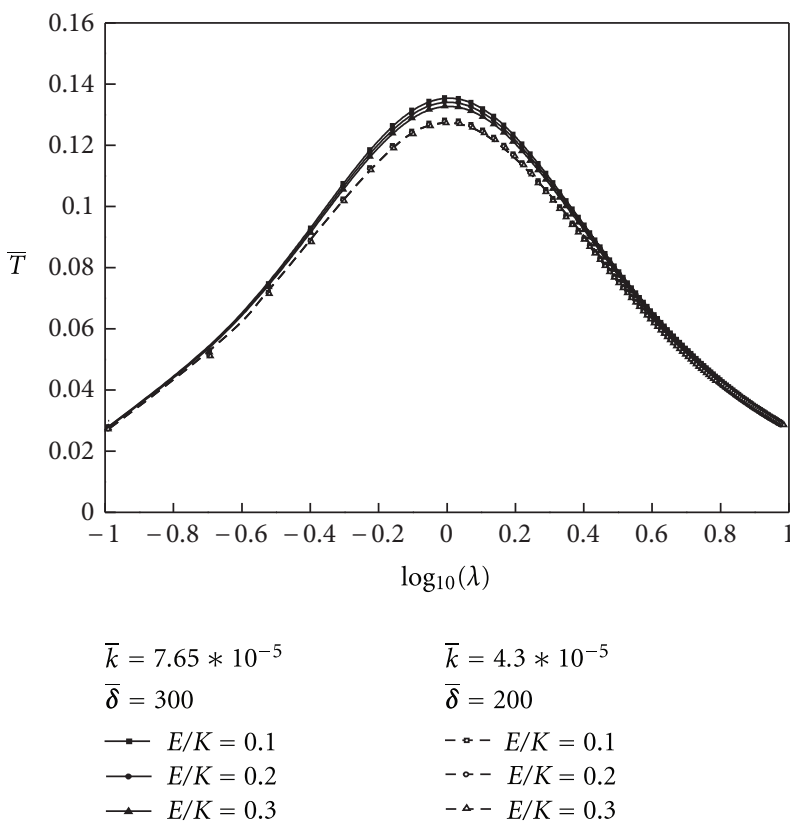

FIGURE 8: Variation of non-dimensional squeeze film time $\bar{T}$ with $\log _{10}(\lambda)$ for different values of $E / K$ with $N=0.2, \bar{l}=0.2, \bar{c}=0.2$.

\section{Results and Discussion}

A simplified mathematical model has been developed for understanding combined effects of surface roughness, poroelasticity, and micropolar fluid on lubrication aspects of synovial joints. The governing equations along with the appropriate constitutive relationships and boundary conditions have been formulated for modeling the roughness structure of cartilage with viscous fluid in the lubricant region in synovial joint lubrication. The load capacity $\bar{W}$ and time height relation $\bar{T}$ are functions of non-dimensional parameters $\bar{c}(=$ $\left.\left(c / h_{0}\right)\right), \psi\left(=\left(k^{*} \delta / h_{0}^{3}\right)\right), \bar{h}=\left(h / h_{0}\right)$. The values of parameters $E / K(=0.1,0.2,0.3), \bar{k}\left(=7.65 \times 10^{-5}, 4.3 \times 10^{-5}\right), \bar{\delta}(=$ $300,200)$ are taken from Torzilli, which are associated with healthy human articular cartilage during normal functioning.

4.1. Load Carrying Capacity. The variations of nondimensional load carrying capacity $\bar{W}$ with aspect ratio $\lambda$ are depicted in Figure 2 for different values of the coupling number $N$ and two values of permeability parameter $\bar{k}$. The curves corresponding to $N=0$ represent the Newtonian case. It is observed that the non-dimensional load carrying capacity increases for the increasing value of a coupling number $N$. Figure 3 shows the variations of $\bar{W}$ with $\log _{10} \lambda$ for different values of $\bar{l}$ with two values of permeability parameter $\bar{k}$. It is observed that $\bar{W}$ increases for increasing values of $\bar{l}$ further; it is also observed that the maximum load carrying capacity is attained for the square plates $(\lambda=1)$.

The effect of elastic parameter $E / K$ on variations of $\bar{W}$ with $\log _{10} \lambda$ is shown in Figure 4 for two values of permeability

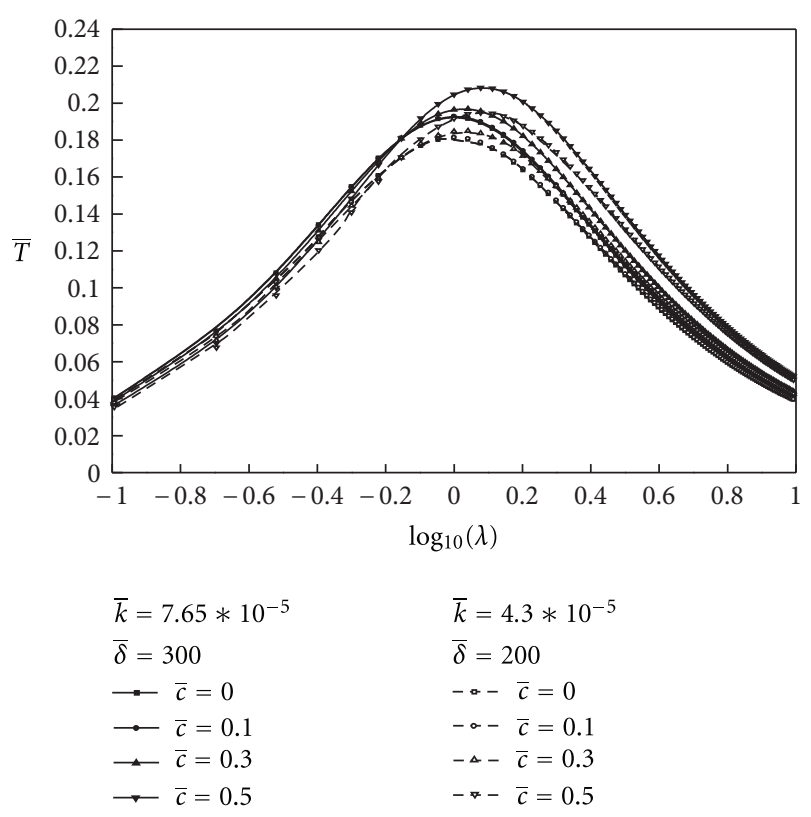

FIgURE 9: Variation of non dimensional squeeze film time $\bar{T}$ with $\log _{10}(\lambda)$ for different values of $\bar{c}$ with $N=0.6, \bar{l}=0.2, E / K=0.3$.

parameter $\bar{k}$. It is observed that $\bar{W}$ increases with $\log _{10} \lambda$ and decreases for increasing values of $E / K$.

The effect of roughness parameter $\bar{c}$ on the variations of $\bar{W}$ with $\log _{10} \lambda$ is depicted in Figure 5 . for two values of permeability parameter $\bar{k}$. It is observed that $\bar{W}$ increases for the increasing values of $\bar{c}$. Further, it is interesting to note that the maximum load carrying capacity $\bar{W}_{\max }$ is a function of $\lambda$ and is obtained for the rectangular plates.

4.2. Squeeze Film Time. The variations of non-dimensional squeeze film time $\bar{T}$ with the aspect ratio $\lambda$ for different values of coupling number $N$ are depicted in the Figure 6 for two values of permeability parameter $\bar{k}$. The curves corresponding to $N=0$ represent the Newtonian case. It is observed that $\bar{T}$ increases for increasing value of $N$.

Figure 7 shows the variation of squeeze film time $\bar{T}$ with $\log _{10} \lambda$ for different values of $\bar{l}$ with two values of permeability parameter $\bar{k}$. The curves corresponding to $\bar{l}=0$ represent the Newtonian case. It is observed that $\bar{T}$ increases for the increases in values of $\bar{l}$. Hence the squeeze film bearings lubricated with micropolar fluid carry larger load for a longer time as compared to the corresponding Newtonian fluids, by which the performance of the bearings is improved.

The effect of elastic parameter $E / K$ on variations of $\bar{T}$ with $\log _{10} \lambda$ is shown in Figure 8 for two values of permeability parameter $\bar{k}$. It is observed that $\bar{T}$ increases with $\log _{10} \lambda$ and decreases for increasing values of $E / K$. The effect of roughness parameter $\bar{c}$ on the variations of $\bar{T}$ with $\log _{10} \lambda$ is depicted in Figure 9. It is observed that $\bar{T}$ increases for the increasing value of $\bar{c}$. 


\section{Conclusion}

The effect of surface roughness on the squeeze film characteristics of rough poroelastic rectangular plates is presented. On the basis of Eringen's micropolar fluid theory and the Christensen stochastic theory for the study of rough surfaces, the modified form of stochastic Reynolds equation is derived for one-dimensional longitudinal roughness pattern. As the micropolar fluid parameter $N \rightarrow 0$ and $\bar{l} \rightarrow 0$, the squeeze film characteristics reduce to corresponding Newtonian case and as $\bar{k} \rightarrow 0$ these characteristics reduce to the smooth case. On the basis of the results presented, the following conclusions are drawn.

(1) The effect of micropolar fluid provides an increased load carrying capacity and squeeze film time as compared to the corresponding Newtonian case.

(2) The effect of surface roughness on the cartilage surface increases the load carrying capacity and squeeze film time as compared to smooth case.

Hence in a practical situation the required shape of the bearing may be rectangular, in which case a specific choice of $\bar{k}, N, \bar{l}$, and $E / K$ will yield larger load carrying capacity and longer squeeze film time as compared to the corresponding Newtonian fluids, by which the performance of the bearings is improved.

\section{Acknowledgments}

The authors are grateful to the referees for their valuable comments on the earlier version of the paper. G. Savitramma also acknowledges the financial support under the Research Fellowship in Science for Meritorious Students (RFSMS) by the University Grants Commission, New Delhi, India.

\section{References}

[1] A. F. Cooke, D. Dowson, and V. Wright, "The rheology of synovial fluid and some potential synthetic lubricants for degenerate synovial joints," Engineering in Medicine, vol. 7, no. 2, pp. 66-72, 1978 .

[2] D. Dowson and J. Zhong-Min, "Micro-elastohydrodynamic lubrication of synovial joints," Engineering in Medicine, vol. 15, no. 2, pp. 63-65, 1986.

[3] V. C. Mow and W. M. Lai, "Recent developments in synovial joints biomechanics," SIAM Review, vol. 22, no. 3, pp. 275-317, 1980.

[4] H. Forster and J. Fisher, "The influence of loading time and lubricant on the friction of articular cartilage," Proceedings of the Institution of Mechanical Engineers, Part H, vol. 210, no. 2, pp. 109-118, 1996.

[5] G. A. Ateshian, "A theoretical formulation for boundary friction in articular cartilage," Journal of Biomechanical Engineering, vol. 119, no. 1, pp. 81-86, 1997.

[6] M. A. Soltz and G. A. Ateshian, "Experimental verification and theoretical prediction of cartilage interstitial fluid pressurization at an impermeable contact interface in confined compression," Journal of Biomechanics, vol. 31, no. 10, pp. 927-934, 1998.
[7] S. Graindorge, W. Ferrandez, E. Ingham, Z. Jin, P. Twigg, and J. Fisher, "The role of the surface amorphous layer of articular cartilage in joint lubrication," Proceedings of the Institution of Mechanical Engineers, Part H, vol. 220, no. 5, pp. 597-607, 2006.

[8] K. C. Morrell, W. A. Hodge, D. E. Krebs, and R. W. Mann, "Corroboration of in vivo cartilage pressures with implications for synovial joint tribology and osteoarthritis causation," Proceedings of the National Academy of Sciences of the United States of America, vol. 102, no. 41, pp. 14819-14824, 2005.

[9] R. Krishnan, E. N. Mariner, and G. A. Ateshian, "Effect of dynamic loading on the frictional response of bovine articular cartilage," Journal of Biomechanics, vol. 38, no. 8, pp. 1665-1673, 2005.

[10] J. E. Pickard, E. Ingham, J. Egan, and J. Fisher, "Investigation into the effect of proteoglycan molecules on the tribological properties of cartilage joint tissues," Proceedings of the Institution of Mechanical Engineers, Part H, vol. 212, no. 3, pp. 177-182, 1998.

[11] P. Kumar, M. Oka, J. Toguchida et al., "Role of uppermost superficial surface layer of articular cartilage in the lubrication mechanism of joints," Journal of Anatomy, vol. 199, no. 3, pp. 241-250, 2001.

[12] I. M. Basalo, F. H. Chen, C. T. Hung, and G. A. Ateshian, "Frictional response of bovine articular cartilage under creep loading following proteoglycan digestion with chondroitinase ABC," Journal of Biomechanical Engineering, vol. 128, no. 1, pp. 131-134, 2006.

[13] J. Katta, T. Stapleton, E. Ingham, Z. M. Jin, and J. Fisher, "The effect of glycosaminoglycan depletion on the friction and deformation of articular cartilage," Proceedings of the Institution of Mechanical Engineers, Part H, vol. 222, no. 1, pp. 1-11, 2008.

[14] R. S. Sayles, T. R. Thomas, and J. Anderson, "Measurement of the surface microgeometry of articular cartilage," Journal of Biomechanics, vol. 12, no. 4, pp. 257-267, 1979.

[15] H. Christensen, "Stochastic models for hydrodynamic lubrication of rough surfaces," vol. 18, pp. 1013-1026, 1969.

[16] N. B. Naduvinamani, S. T. Fathima, and P. S. Hiremath, "On the squeeze effect of lubricants with additives between rough porous rectangular plates," Zeitschrift für Angewandte Mathematik und Mechanik, vol. 84, no. 12, pp. 825-834, 2004.

[17] V. K. Agrawal, K. L. Ganju, and S. C. Jethi, "Squeeze film and externally pressurized bearings micro-polar fluid lubricated," Wear, vol. 19, no. 3, pp. 259-265, 1972.

[18] N. M. Bujurke, S. G. Bhavi, and P. S. Hiremath, "Squeeze film lubri-cated with micropolar fluids," Proceedings of Indian National Science Academy, vol. 53, no. 3, pp. 391-398, 1987.

[19] A. C. Eringen, “Theory of micropolar fluids," Journal of Applied Mathematics and Mechanics, vol. 16, pp. 1-18, 1966.

[20] P. A. Torzilli and V. C. Mow, "On the fundamental fluid transport mechanisms through normal and pathological articular cartilage during function. II. The analysis, solution and conclusions," Journal of Biomechanics, vol. 9, no. 9, pp. 587-606, 1976.

[21] R. Collins, "A model of lubricant gelling in synovial joints-part I: derivation and properties of mathematical model," Zeitschrift für Angewandte Mathematik und Physik, vol. 33, no. 1, pp. 93-123, 1982.

[22] R. Y. Hori and L. F. Mockros, "Indentation tests of human articular cartilage," Journal of Biomechanics, vol. 9, no. 4, pp. 259-268, 1976. 
[23] P. Sinha and C. Singh, "Micropolar squeeze films between rough rectangular plates," Applied Scientific Research, vol. 39, no. 3, pp. 167-179, 1982.

[24] H. Wu, "Analysis of the squeeze film between porous rectangular plates," ASME Transactions Journal of Lubrication Technology, vol. 94, no. 1, pp. 64-68, 1972. 

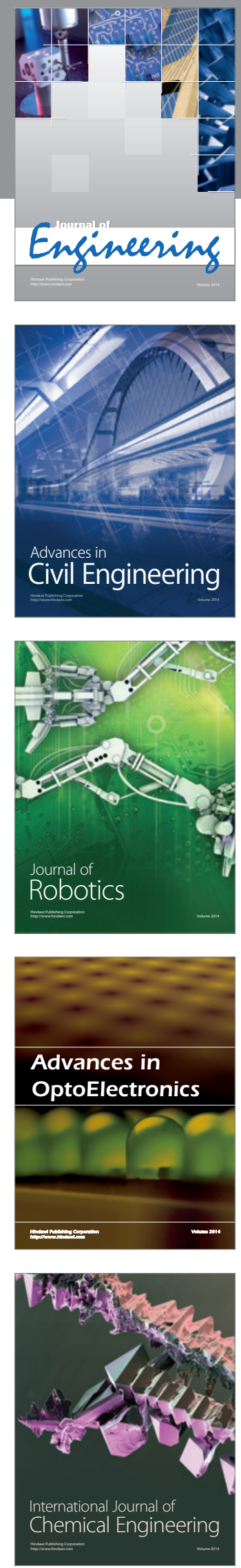

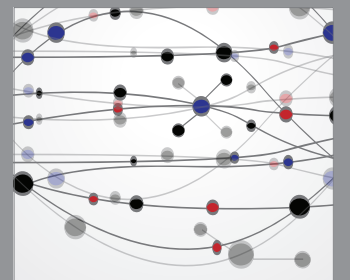

The Scientific World Journal
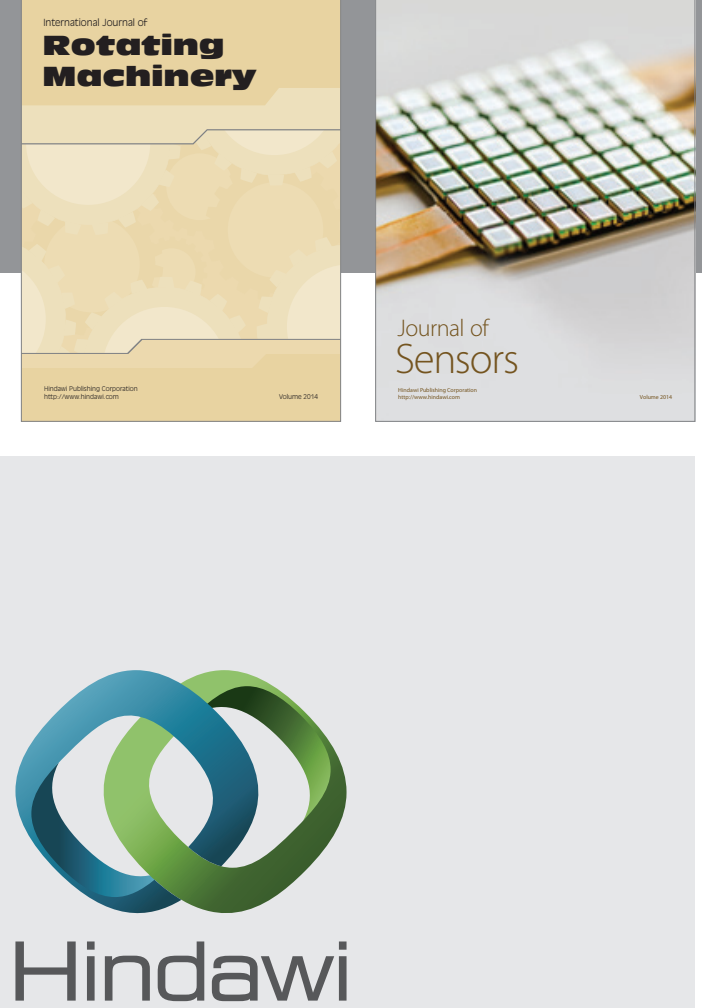

Submit your manuscripts at http://www.hindawi.com
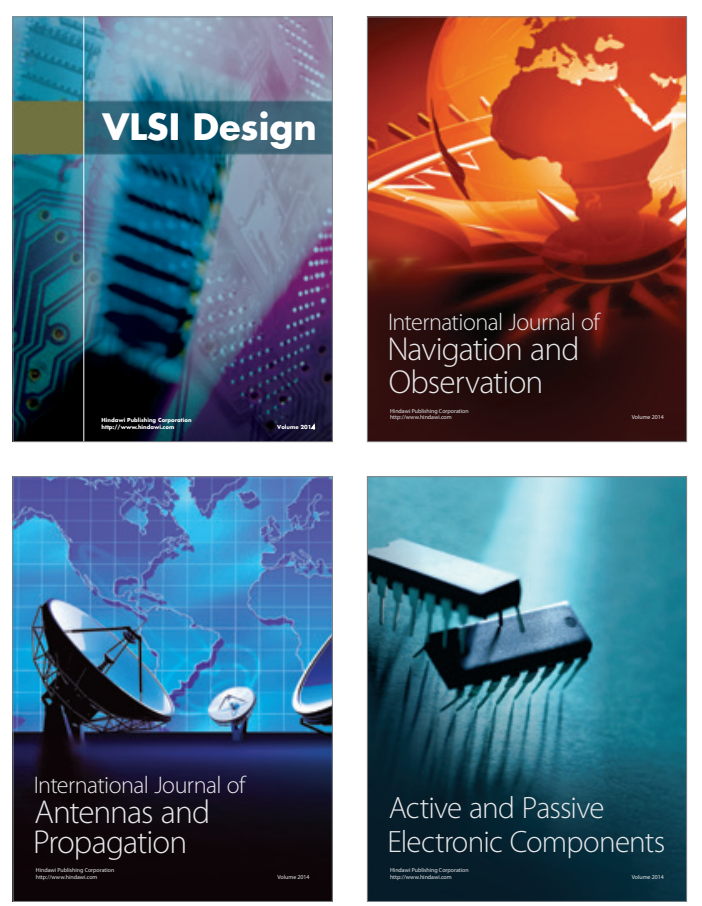
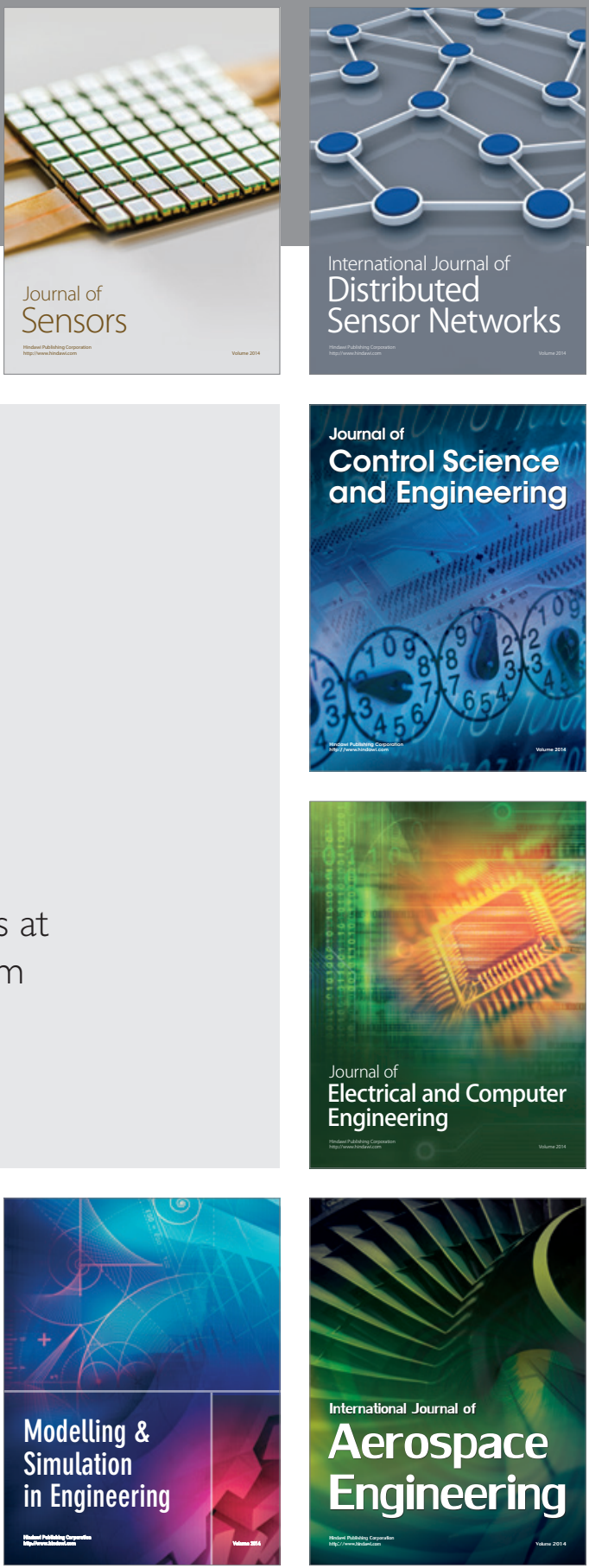

Journal of

Control Science

and Engineering
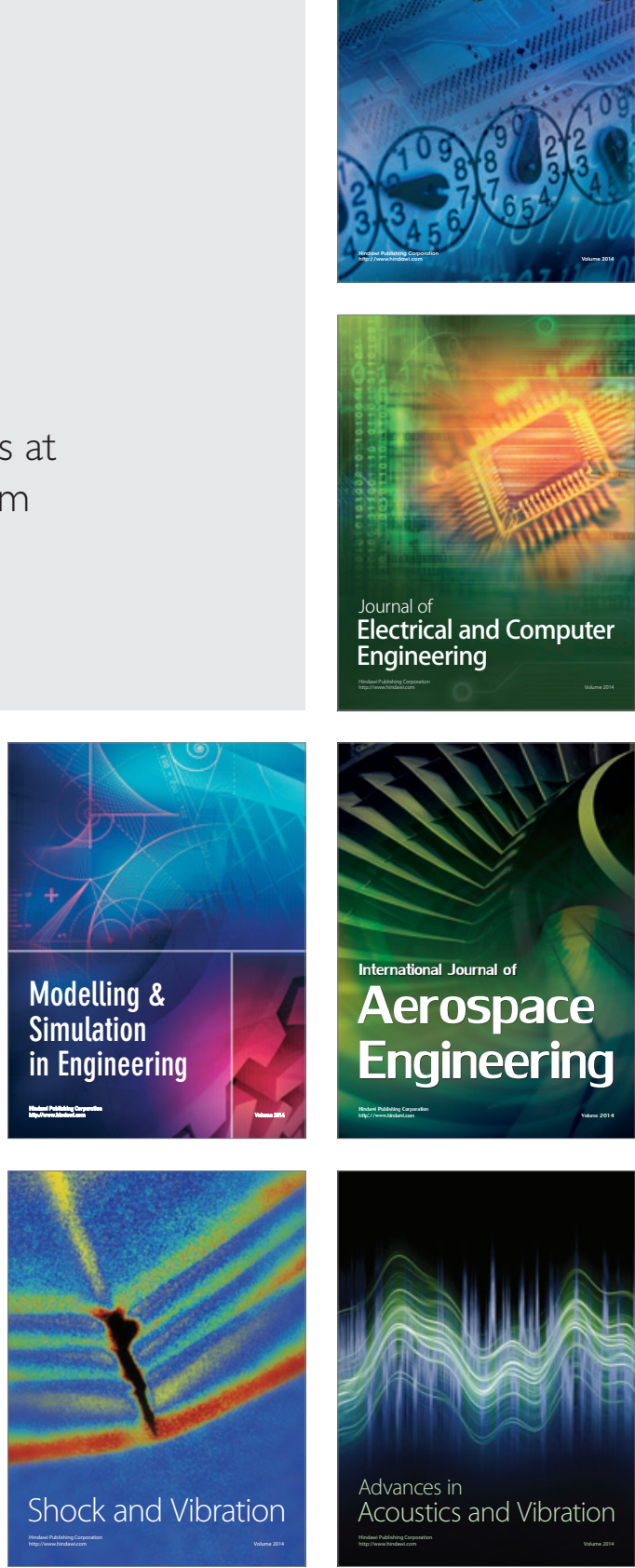